\title{
Molecular single-particle excitations in heavy-ion reactions involving deformed light nuclei
}

\author{
Jae Young Park \\ Department of Physics, North Carolina State University, Raleigh, North Carolina 27650
}

Werner Scheid

Institut für Theoretische Physik der Justus-Liebig-Universität,

Giessen, Germany

Walter Greiner

Institut für Theoretische Physik der Johann-Wolfgang-Goethe-Universität, Frankfurt am Main, Germany

(Received 9 October 1981)

Two-center level diagrams for the neutron orbitals in the scattering of ${ }^{16} \mathrm{O}$ on ${ }^{25} \mathrm{Mg}$ and of ${ }^{17} \mathrm{O}$ on ${ }^{24} \mathrm{Mg}$ are calculated by using a deformed potential for ${ }^{24,25} \mathrm{Mg}$. Possible consequences of the nuclear Landau-Zener mechanism, namely the promotion of nucleons at avoided level crossings, and of the rotational coupling between crossing molecular singleparticle orbitals are studied for inelastic excitation and neutron transfer. The important excitation and transfer processes, which are enhanced by the promotion process and the rotational coupling, are presented.

$\left[\begin{array}{c}\text { NUCLEAR REACTIONS Heavy ion scattering, theory of nucleon } \\ \text { transfer, molecular wave functions, asymmetric two center shell model, } \\ \text { single particle excitation, deformed nuclei. }\end{array}\right]$

\section{INTRODUCTION}

One of the exciting results of heavy-ion physics is the possibility that nuclear molecules ${ }^{1}$ can be formed during the scattering process. In the microscopic description of nuclear molecules one assumes that the outermost loosely bound nucleons orbit around both nuclear centers and generate a homopolar or covalent binding. Molecular singleparticle effects are not well established yet, even though collective molecular resonances have been observed in several heavy-ion systems, such as ${ }^{12} \mathrm{C}+{ }^{12} \mathrm{C}$ and ${ }^{12} \mathrm{C}+{ }^{16} \mathrm{O}$ (for details see Ref. 1 ).

Whether molecular orbitals are formed depends on the ratio of two characteristic times, the collision time $\tau_{c} \sim 2 R / v=2 R(2 E / M A)^{-1 / 2}$ and the nuclear period or single-particle relaxation time $\tau_{s} \gtrsim$ $2 R / v_{\text {Fermi }}=2 R\left(2 \epsilon_{F} / M\right)^{-1 / 2}$, where $E$ is the laboratory bombarding energy, $A$ the projectile mass number, and $\epsilon_{F}$ the Fermi energy in the target or projectile nucleus with valence nucleons. In typical low-energy collisions this ratio is

$$
\tau_{c} / \tau_{s} \sim \sqrt{\epsilon_{F} /(E / A)} \sim \sqrt{30 / 5} \sim 2.4
$$

According to this ratio we expect that molecular orbitals and the polarization of valence nucleons by the field of the other nucleus may have time to develop.

Recently, new experimental works to look for evidence of molecular orbital phenomena have been carried out. For example, the Yale group observed resonantlike behavior in the excitation functions of the ${ }^{13} \mathrm{C}\left({ }^{12} \mathrm{C},{ }^{12} \mathrm{C}\right){ }^{14} \mathrm{C}$ single-nucleon transfer reactions. ${ }^{2}$ From DWBA calculations, such pronounced structure is unlikely. Indeed it was found that DWBA predicts little structure and is unsatisfactory in reproducing oscillations in the angular distributions. $^{2}$ Structure observed in the energy dependence of the cross section indicates that some interesting processes, such as molecular orbital phenomena, may be occurring. A recent coupled channel calculation by von Oertzen et al. ${ }^{3}$ confirms that the neutron orbits in the grazing collision of ${ }^{12} \mathrm{C}$ on ${ }^{13} \mathrm{C}$ are of molecular nature and this prediction is 
supported by their experiment. ${ }^{3}$ Imanishi et $a l^{4}$ have shown also in the case of ${ }^{16} \mathrm{O}$ scattering on ${ }^{17} \mathrm{O}$ leading to the excited state of ${ }^{17} \mathrm{O}^{*}\left(0.87 \mathrm{MeV}, \frac{1}{2}^{+}\right)$ that the neutron can be transferred back and forth at least twice, thus indicating the formation of molecular orbitals. In Ref. 5 we examined the possibility of the nuclear Landau-Zener excitation mechanism, namely the promotion of nucleons at level crossings, which may cause specific structures in the energy dependence of the heavy-ion cross sections. Specifically, in the previous work, ${ }^{5}$ we calculated level diagrams of the two-center shell model for the ${ }^{13} \mathrm{C}+{ }^{16} \mathrm{O}$ and ${ }^{12} \mathrm{C}+{ }^{17} \mathrm{O}$ systems and discussed the possible consequences of level crossings for inelastic excitation and neutron transfer.

In the present work, we extend our consideration for asymmetric heavy-ion collisions involving light deformed nuclei. We present calculations of the two-center level structure for the ${ }^{16} \mathrm{O}+{ }^{25} \mathrm{Mg}$ and ${ }^{17} \mathrm{O}+{ }^{24} \mathrm{Mg}$ systems and discuss the possible important reactions involving the excitation and the transfer of a neutron. In this calculation the ${ }^{25} \mathrm{Mg}$ nucleus is described with an intrinsic deformation built into the potential of the asymmetric twocenter shell model ${ }^{6}$ (ATCSM). For large internuclear distances the ATCSM yields a single-particle spectrum for ${ }^{25} \mathrm{Mg}$ which is in accordance with the spectrum obtained in the Nilsson model. The choice of the parameters of the ATCSM and the calculation of the level diagrams are presented in Sec. II.

A systematic study of two-center level diagrams leads to an understanding of the possible molecular single-particle effects without going into the details of coupled channel calculations. Realistic twocenter level diagrams are valuable in predicting all the reactions which should be enhanced by the promotion of nucleons at internuclear distances, where avoided crossings of molecular single-particle levels occur. Possible reactions of this type will be discussed in Sec. III for ${ }^{16} \mathrm{O}+{ }^{25} \mathrm{Mg}$ and ${ }^{17} \mathrm{O}+{ }^{24} \mathrm{Mg}$ on the basis of the two-center level diagrams obtained in Sec. II.

\section{CALCULATION OF LEVEL DIAGRAMS USING THE ASYMMETRIC TWO-CENTER SHELL MODEL}

In order to study Landau-Zener effects in heavyion reactions involving deformed light nuclei we have calculated single-particle energies as a function of the internuclear distance for the systems ${ }^{16} \mathrm{O}+$
${ }^{25} \mathrm{Mg}$ and ${ }^{17} \mathrm{O}+{ }^{24} \mathrm{Mg}$. In this calculation we used the asymmetric two-center shell model (ATCSM) of Maruhn and Greiner. ${ }^{6}$ The potential of the ATCSM along the $z$ axis and the associated nuclear shape are shown in Fig. 1. The deformation parameters $\beta_{i}$ of the ATCSM are defined by the ratio of the axes (see Fig. 1)

$\beta_{i}=a_{i} / b_{i}$, for $i=1,2$.

The barrier parameter $\epsilon$ is defined by the ratio of the actual barrier height to that of the asymmetric two-center oscillator (Fig. 1):

$$
\epsilon=E_{0} / E^{\prime}
$$

In all calculations we used the empirical value $\epsilon=0.68$ which was found in Ref. 5 to give a proper asymptotic convergence of the magnetic sublevels. The parameters of the two-center potential are determined by fitting the experimental singleparticle energies of the separated nuclei $(R=\infty)$ and the corresponding composite nucleus $(R=0)$ near the Fermi surface.

\section{A. The parameters of the ATCSM potential for $R=0$ and $R=\infty$}

For zero deformations $\left(\beta_{i}=1\right)$ and internuclear distances $R=0$ and $R=\infty$ the ATCSM yields the
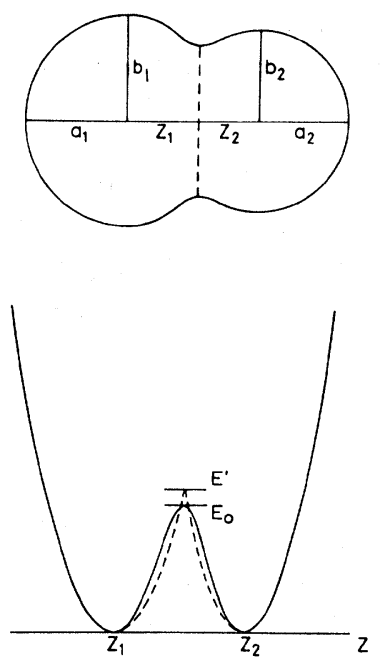

FIG. 1. The potential of the ATCSM along the $z$ axis and the associated nuclear shape. 
single-particle spectrum of a shell model with a spherical oscillator potential. The corresponding single-particle energies can be written as follows:

$$
\begin{aligned}
\epsilon(N l j) & =\left(N+\frac{3}{2}\right) \hbar \omega_{0} \\
& +\frac{\widetilde{a}}{2}\left[j(j+1)-l(l+1)-\frac{3}{4}\right] \\
& +\widetilde{b}\left[l(l+1)-\frac{1}{2} N(N+3)\right]-V_{0},
\end{aligned}
$$

with $\hbar \omega_{0}=C / A^{1 / 3}$. The parameters $\widetilde{a}$ and $\widetilde{b}$ are the strengths of the spin orbit and the $l^{2}$-type interactions. For the spherical nuclei ${ }^{16} \mathrm{O},{ }^{17} \mathrm{O}$, and ${ }^{41} \mathrm{Ca}$ we have used Eq. (3) to fit the parameters $C$, $V_{0}, \widetilde{a}$, and $\widetilde{b}$ with the experimental single-particle energies listed in Table I.

The ${ }^{25} \mathrm{Mg}$ nucleus has an intrinsic quadrupole moment of $Q_{0}=0.616 \times 10^{2} \mathrm{fm}^{2}$ (Ref. 7). For a spheroidal shape with semi-axes, $a$ and $b$, the electric quadrupole moment is given by

$$
Q_{0}=\frac{2}{5} Z\left(a^{2}-b^{2}\right)
$$

Introducing the radius $R_{0}$ of a sphere with the same volume as the spheroid $\left(a b^{2}=R_{0}{ }^{3}\right)$, and using $\beta=a / b$ [see Eq. (1)] we obtain

$$
Q_{0}=\frac{2}{5} Z R_{0}^{2} \beta^{-2 / 3}\left(\beta^{2}-1\right) \text {. }
$$

For the experimental value of $Q_{0}$ and with $R_{0}=$ $1.25 A^{1 / 3} \mathrm{fm}$ we get $\beta=1.50$. This value of the ratio of axes corresponds to a quadrupole deformation of $\beta_{2}=0.45$. Because of this large value of the deformation parameter we have calculated the singleparticle energies of ${ }^{24} \mathrm{Mg}$ and ${ }^{25} \mathrm{Mg}$ in the fitting procedure numerically using the ATCSM program.

Figure 2 shows the single-particle levels of ${ }^{24} \mathrm{Mg}$ as a function of the ratio $\beta$ of axes for fixed values of $C, \widetilde{a}, \widetilde{b}$, and $V_{0}$. The "experimental" single-

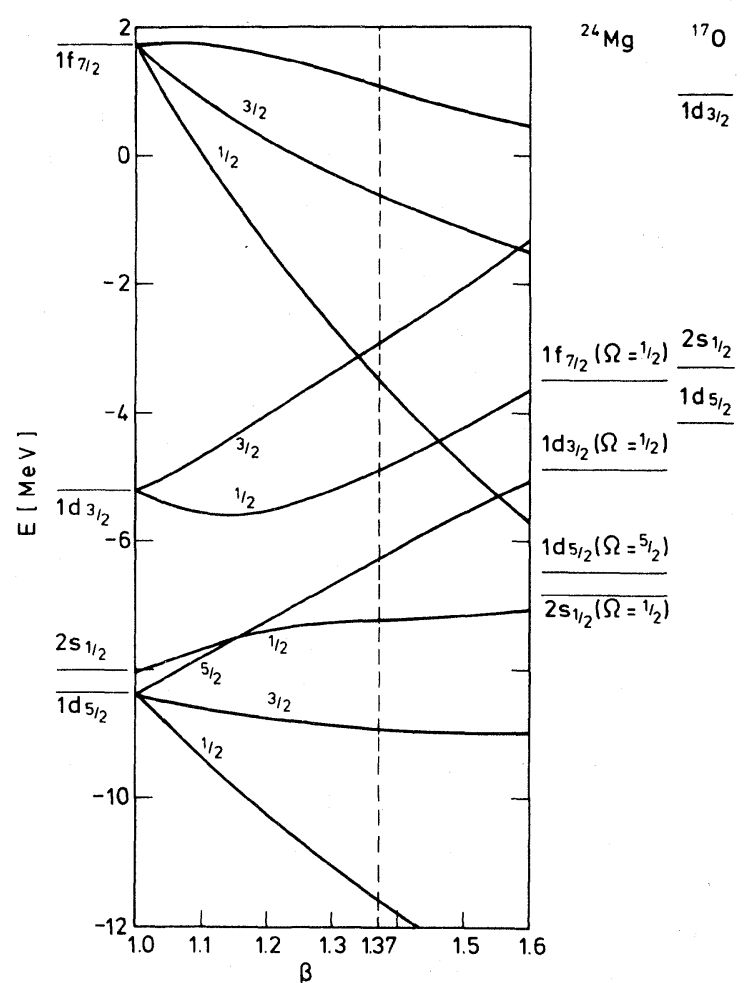

FIG. 2. The single-particle energies of ${ }^{24} \mathrm{Mg}$ as a function of the deformation parameter $\beta$ defined by Eq. (1). The energies are calculated with the ATCSM for $R=20$ $\mathrm{fm}$ with the parameters listed in Table III. For comparison the "experimental" single-particle energies of ${ }^{24} \mathrm{Mg}$ are depicted. Also the experimental single-particle energies of ${ }^{17} \mathrm{O}$ are shown.

particle levels, also shown in Fig. 2, are obtained from the spectrum of ${ }^{25} \mathrm{Mg}$ as follows: The spectrum of ${ }^{25} \mathrm{Mg}$ can be explained by the strong coupling of the neutron motion to the deformed ${ }^{24} \mathrm{Mg}$ core and described in the framework of the Nilsson model and the aligned coupling scheme., ${ }^{8,9}$ The ${ }^{25} \mathrm{Mg}$ states can be grouped into rotational bands built on single-particle excitations. The energies of the levels are given by the formula ${ }^{10,11}$

$$
E_{I, K}=\epsilon_{\Omega=K}+\frac{\hbar^{2}}{2 J}\left[I(I+1)-2 K^{2}+\delta_{K, 1 / 2} d(-1)^{I+1 / 2}(I+1 / 2)\right]
$$

TABLE I. Experimental neutron single-particle energies (in $\mathrm{MeV}$ ) of ${ }^{17} \mathrm{O}$ (Ref. 15) and ${ }^{41} \mathrm{Ca}$ (Ref. 7).

\begin{tabular}{lcccccr}
\hline \hline Level: & $1 d_{5 / 2}$ & $2 s_{1 / 2}$ & $1 d_{3 / 2}$ & $1 f_{7 / 2}$ & $2 p_{3 / 2}$ & $2 p_{1 / 2}$ \\
\hline${ }^{17} \mathrm{O}$ & -4.14 & -3.27 & 0.94 & & & \\
${ }^{41} \mathrm{Ca}$ & & & & -8.36 & -6.26 & -4.46 \\
\hline \hline
\end{tabular}


TABLE II. The characteristic quantities of the lowest rotational bands of ${ }^{25} \mathrm{Mg}$ (from Ref. 11): the energies of the band heads, measured with respect to the ground state and to the negative value of the neutron separation energy $(-7.332 \mathrm{MeV})$, their spin $I$, spin projection $K$ on the body-fixed symmetry axis, and parity $\pi$, the inertia parameter $\hbar^{2} / 2 J$ of the rotational energy and decoupling parameter $d$, the single particle energies $\epsilon_{\Omega}$, calculated according to Eq. (6), and the quantum numbers of the corresponding spherical states for zero deformation.

\begin{tabular}{lcrrr}
\hline \hline$E_{I K}^{*}(\mathrm{MeV})$ & 0 & 0.585 & 2.562 & 3.414 \\
$E_{I K}(\mathrm{MeV})$ & -7.332 & -6.747 & -4.770 & -3.918 \\
$\quad I, K, \pi$ & $\frac{5}{2}, \frac{5}{2},+$ & $\frac{1}{2}, \frac{1}{2},+$ & $\frac{1}{2}, \frac{1}{2},+$ & $\frac{3}{2}, \frac{1}{2},-$ \\
$\hbar^{2} / 2 J(\mathrm{MeV})$ & 0.231 & 0.163 & 0.150 & 0.113 \\
$d(\mathrm{MeV})$ & & -0.202 & -0.473 & -3.54 \\
$\epsilon_{\Omega=K}(\mathrm{MeV})$ & -6.47 & -6.82 & -4.88 & -3.48 \\
Spherical & $1 d_{5 / 2}$ & $2 s_{1 / 2}$ & $1 d_{3 / 2}$ & $1 f_{7 / 2}$ \\
$\quad$ state & & & & \\
\hline \hline
\end{tabular}

Here, $\epsilon_{\Omega}$ is the single-particle energy, $d$ the decoupling parameter, and $K$ and $\Omega$ the components of the total and single-particle angular momenta along the body-fixed axis, respectively. In order to obtain the absolute values of the single-particle energies we have set the energy of the ground state of ${ }^{25} \mathrm{Mg}$ to be equal to the negative value of the neutron separation energy, i.e., $E_{I=5 / 2, K=5 / 2}=-7.332 \mathrm{MeV}$. The single-particle energies are obtained according to Eq. (6) by using the experimental energies $E_{l, K}$ and the values of $\hbar / 2 J$ and $d$ given in Table II. These "experimental" single-particle energies, listed in Table II, can be specified by the quantum numbers of the states in a spherical potential, to which they approach for zero deformation.

Comparing the "experimental" single-particle energies with the calculated ones (see Fig. 2) we conclude that a realistic order of levels near the Fermi surface can be obtained for $\beta=1.37$. In this case the energies of the levels $1 f_{7 / 2}\left(\Omega=\frac{1}{2}\right)$ and $1 d_{3 / 2}$ $\left(\Omega=\frac{1}{2}\right)$ are fitted exactly. The levels $1 d_{5 / 2}\left(\Omega=\frac{5}{2}\right)$ and $2 s_{1 / 2}\left(\Omega=\frac{1}{2}\right)$ have the correct order, and their energies agree roughly with the "experimental" ones.

The final parameters of the ATCSM at $R=0$ and $R=\infty$ which we used in the calculations are listed in Table III. The values of $\hbar \omega, \widetilde{a}, \widetilde{b}$, and $V_{0}$ at internuclear distances between these limits are properly interpolated by using the values given in Table III.

$$
\begin{gathered}
\text { B. The level diagrams } \\
\text { for }{ }^{16} \mathrm{O}+{ }^{25} \mathrm{Mg} \text { and }{ }^{17} \mathrm{O}+{ }^{24} \mathrm{Mg}
\end{gathered}
$$

Figures 3 and 4 present the level diagrams calculated with the set of parameters listed in Table III.

TABLE III. The parameters of the ATCSM potential (in MeV) for $R=0$ and $R=\infty$. The parameters $\widetilde{a}$ and $\widetilde{b}$ are the strengths of the spin-orbit and $l^{2}$-type interactions [for definition see Ref. 5 and Eq. (3)].

\begin{tabular}{cccccccc}
\hline \hline System & $C=\hbar \omega_{0} A^{1 / 3}$ & Nucleus & $\hbar \omega_{0}$ & $\tilde{a}$ & $\tilde{b}$ & $V_{0}$ & Fitted levels \\
& & & & & & & \\
& & ${ }^{16} \mathrm{O}$ & 11.90 & -2.898 & 0.338 & 43.23 & $1 d_{5 / 2}, 2 s_{1 / 2}$ \\
${ }^{16} \mathrm{O}+{ }^{25} \mathrm{Mg}$ & 30.0 & ${ }^{25} \mathrm{Mg}$ & 10.26 & -1.260 & 0.165 & 43.23 & $1 d_{3 / 2}\left(\Omega=\frac{1}{2}\right), 1 f_{7 / 2}\left(\Omega=\frac{1}{2}\right)$ \\
& & ${ }^{41} \mathrm{Ca}$ & 8.70 & -1.200 & -0.090 & 45.44 & $1 f_{7 / 2}, 2 p_{3 / 2}, 2 p_{1 / 2}$ \\
& & & & & & & \\
${ }^{17} \mathrm{O}+{ }^{24} \mathrm{Mg}$ & 30.0 & ${ }^{24} \mathrm{O}$ & 11.67 & -1.247 & 0.063 & 43.79 & $1 d_{5 / 2}, 2 s_{1 / 2}$ \\
& & 10.40 & -1.270 & 0.155 & 43.79 & $1 d_{3 / 2}\left(\Omega=\frac{1}{2}\right), 1 f_{7 / 2}\left(\Omega=\frac{1}{2}\right)$ \\
& & ${ }^{41} \mathrm{Ca}$ & 8.70 & -1.200 & -0.090 & 45.44 & $1 f_{7 / 2}, 2 p_{3 / 2}, 2 p_{1 / 2}$ \\
\hline \hline
\end{tabular}




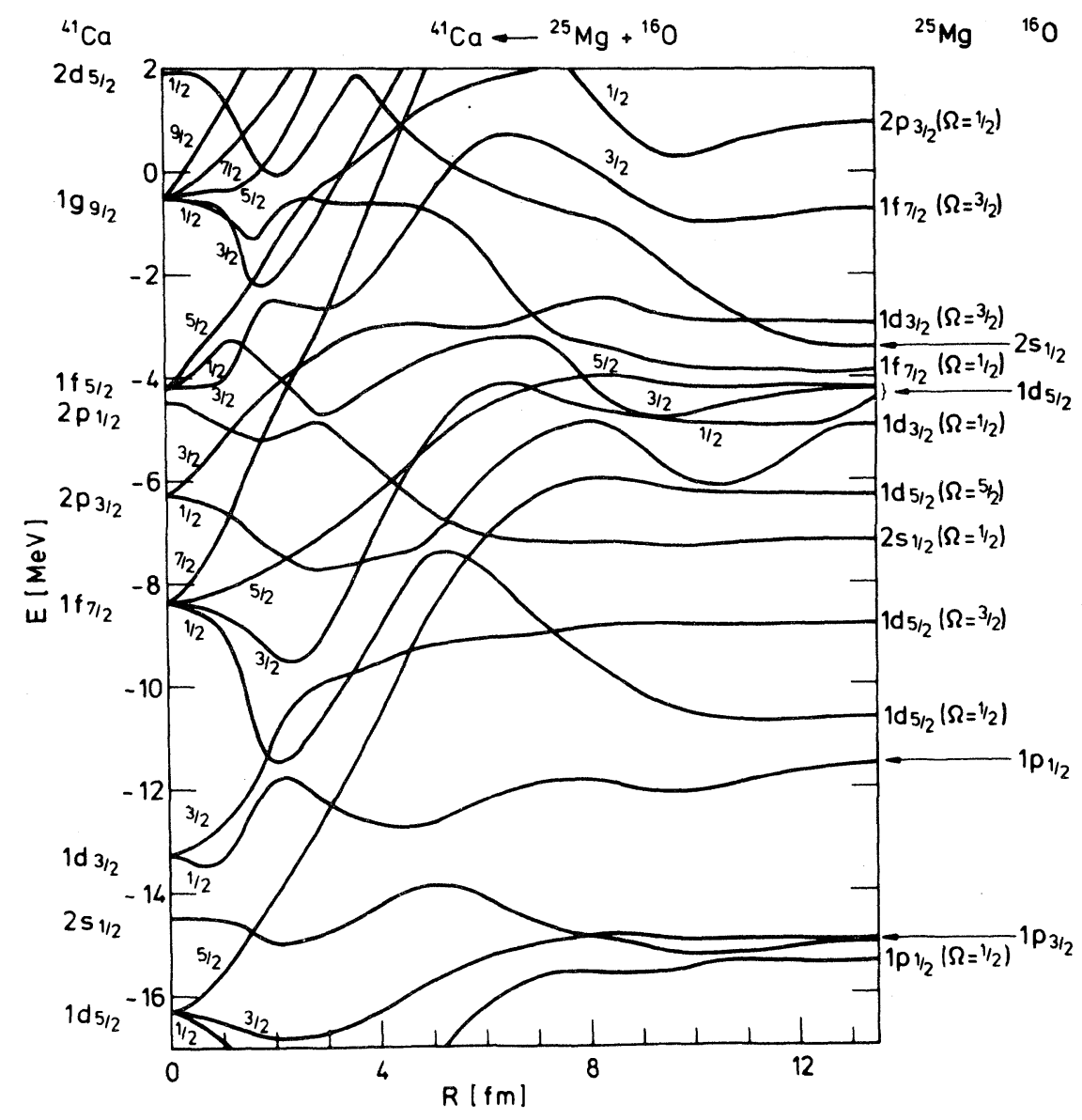

FIG. 3. The neutron level diagram as a function of the internuclear distance $R$, as calculated with the ATCSM for the reaction ${ }^{16} \mathrm{O}+{ }^{25} \mathrm{Mg} \rightarrow{ }^{41} \mathrm{Ca}$. The values of the parameters which are used in the calculations are given in Table III.

It should be noted that the potential of the ATCSM considered by Maruhn and Greiner ${ }^{6}$ is spherically symmetric about the axis connecting the two centers. Therefore, the level diagrams, presented in the figures, correspond to the special case in which the symmetry axis of $\mathrm{Mg}$ lies parallel to the internuclear axis. One expects that the single-particle levels depend on the relative position of the symmetry axis of ${ }^{25} \mathrm{Mg}$ and internuclear axis in the touching zone of the nuclei. In order to treat the dependence of the single-particle levels on the relative position of the nuclear deformations with respect to the internuclear axis, one has to develop a new parametrization of the ATCSM potential which has not yet been considered in literature. Such a new ATCSM potential is needed in dynamical calculations of the scattering of deformed nuclei in the framework of molecular single-particle wave functions.

Since we have fitted the parameters of the
ATCSM with the experimental single-particle levels we have achieved the correct order of the levels in the diagrams near the Fermi level. The correct order of levels is important for the discussion of possible reactions on the basis of level diagrams.

\section{DISCUSSION OF POSSIBLE REACTIONS}

The level diagrams, shown in Figs. 3 and 4, can be used for the discussion of possible reactions. There are two main excitation mechanisms between molecular single-particle states, the radial and rotational couplings. Let $\varphi_{\Omega, \lambda}(\vec{r}, R)$ be the singleparticle wave functions of the ATCSM, where $\Omega$ is the quantum number of the angular momentum component in the direction of the internuclear axis and $\lambda$ the remaining quantum numbers of the states. Then the transition matrix elements of the 


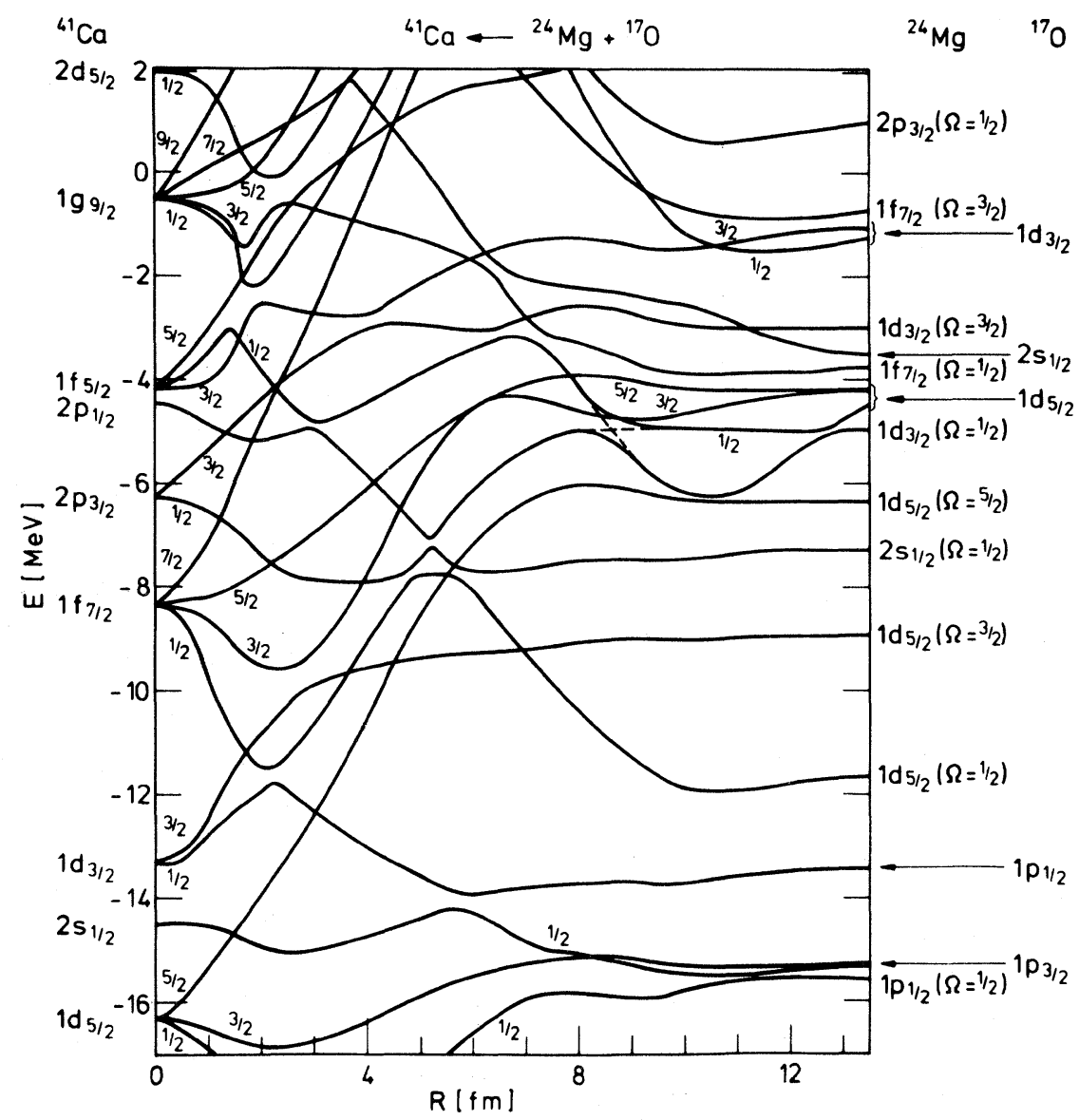

FIG. 4. The neutron level diagram as a function of the internuclear distance $R$, as calculated with the ATCSM for the reaction ${ }^{17} \mathrm{O}+{ }^{24} \mathrm{Mg} \rightarrow{ }^{41} \mathrm{Ca}$.

radial and rotational couplings can be written as:

$$
\left\langle\varphi_{\Omega \lambda}\left|\frac{\partial}{\partial R}\right| \varphi_{\Omega \lambda^{\prime}}\right\rangle
$$

and

$$
\left\langle\varphi_{\Omega \lambda}\left|j_{ \pm}\right| \varphi_{\Omega \mp 1, \lambda^{\prime}}\right\rangle .
$$

Here, $j_{ \pm}=j_{x} \pm i j_{y}$ are the angular momentum operators of the single particle. At avoided crossings of levels with the same value of $\Omega$, the molecular wave functions change rapidly as a function of the internuclear distance. At these distances the matrix elements (7) have the largest values and, therefore, the processes, induced by the radial coupling, are strongly enhanced. According to the Landau-Zener formula, ${ }^{12}$ the probability $P$ of a quantum jump from one level to another one at an avoided crossing (pseudocrossing) at $R_{c}$ is given by

$$
P=e^{-2 \pi G},
$$

where the dimensionless "interaction parameter" $G$ is given by

$$
G=\frac{1}{\hbar v_{c}} \frac{\left|H_{12}\right|^{2}}{\left|\frac{d}{d R}\left[\epsilon_{1}(R)-\epsilon_{2}(R)\right]\right|_{R=R_{c}}}
$$

Here, $H_{12}$ is the matrix element for the coupling between the unperturbed states 1 and 2 with energies $\epsilon_{1}(R)$ and $\epsilon_{2}(R)$, which cross at $R_{c}$ without perturbation. The probability $P$ for excitation increases with increasing relative velocity $v_{c}$ of the two nuclei. In Figs. 3 and 4 we recognize many pairs of levels between which the radial and rotational couplings can cause the excitation and transfer of nucleons. The locations, i.e., the internuclear distances, where these reactions can proceed are indicated in Figs. 5-8. The distances where avoided level crossings occur are fairly independent of varia- 


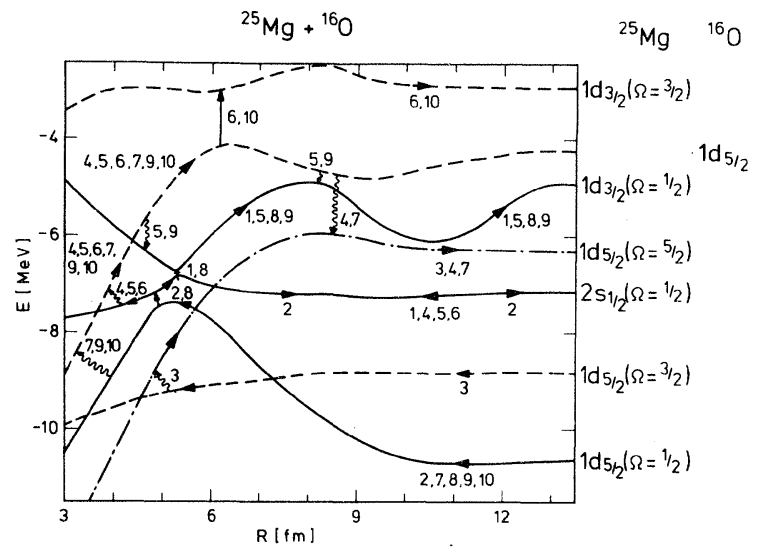

FIG. 5. Inelastic excitation in the reaction ${ }^{16} \mathrm{O}+{ }^{25} \mathrm{Mg}$. The figure illustrates the processes listed in Table IV. The numbers are the process numbers given in Table IV. The solid, dashed, and dashed-dotted lines represent the levels with $\Omega=\frac{1}{2}, \frac{3}{2}$, and $\frac{5}{2}$. The straight and wavy arrows indicate transitions induced by the radial and rotational couplings, respectively.

tions in the parameter sets of the ATCSM, as also found in the previous work. ${ }^{5}$ In the following discussion we will not consider level crossings which are located well inside the interaction region, say $R<3 \mathrm{fm}$, since they are hidden by the centrifugal potentials due to the radial relative motion and by the strong absorption occurring inside. Also we will discuss only one-nucleon excitation and transfer.

\section{A. The ${ }^{16} \mathrm{O}+{ }^{25} \mathrm{Mg}$ reactions}

In Tables IV and $\mathrm{V}$ we have listed possible inelastic and neutron transfer processes which can occur in the scattering of ${ }^{16} \mathrm{O}$ on ${ }^{25} \mathrm{Mg}$ according to the

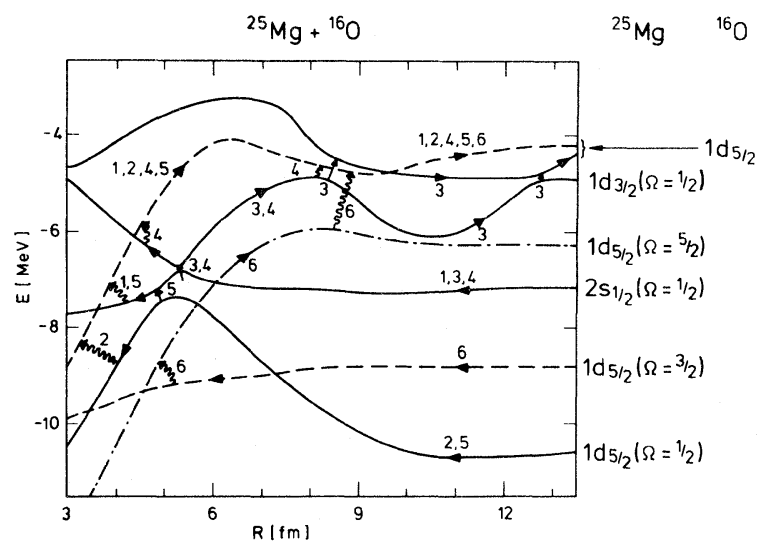

FIG. 6. Transfer of a neutron in the reaction ${ }^{16} \mathrm{O}+{ }^{25} \mathrm{Mg}$. The figure illustrates the processes listed in Table V. For further explanations see the caption of Fig. 5.

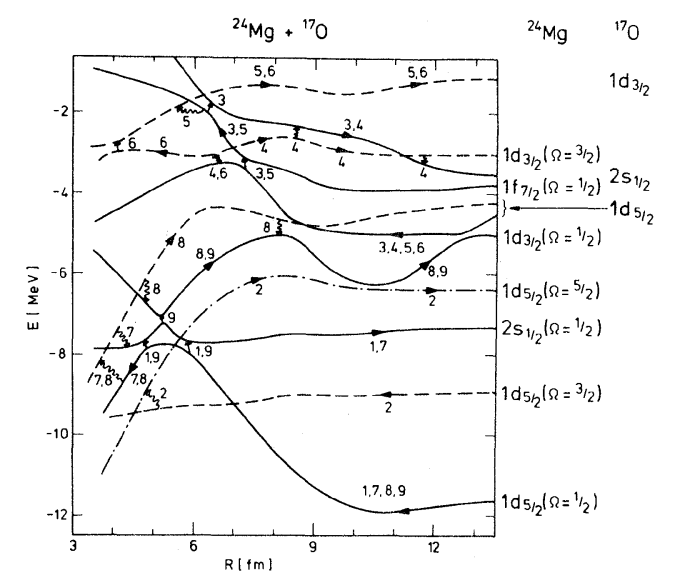

FIG. 7. Inelastic excitation in the reaction ${ }^{17} \mathrm{O}+{ }^{24} \mathrm{Mg}$. The figure illustrates the processes listed in Table VI. For further explanations see the caption of Fig. 5.

level diagram shown in Fig. 3. In all these processes a single neutron is promoted to a higher level by the radial and rotational couplings at localized relative distances. The number of steps, given in the tables, counts the number of localized couplings acting between the initial and final states of the neutron. For simplicity we mainly consider oneand two-step processes.

The tables contain an assignment number for the process, the number of steps, the final and initial states of the neutron, the intermediate state in the case of two step processes, the approximate locations of the couplings, the type of excitation mechanism, and the $\Omega$-quantum numbers of the

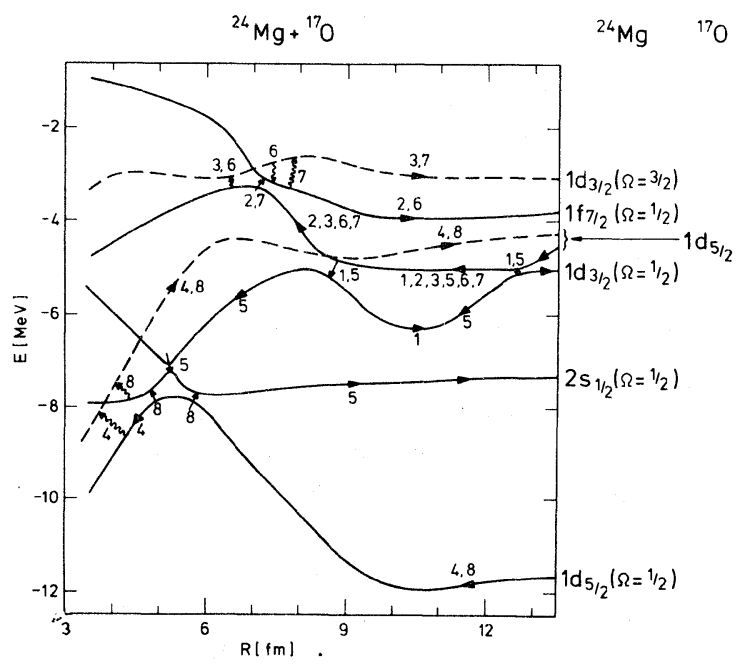

FIG. 8. Transfer of a neutron in the reaction ${ }^{1} \mathrm{O}+{ }^{24} \mathrm{Mg}$. The figure illustrates the processes listed in Table VII. For further explanations see the caption of Fig. 5. 


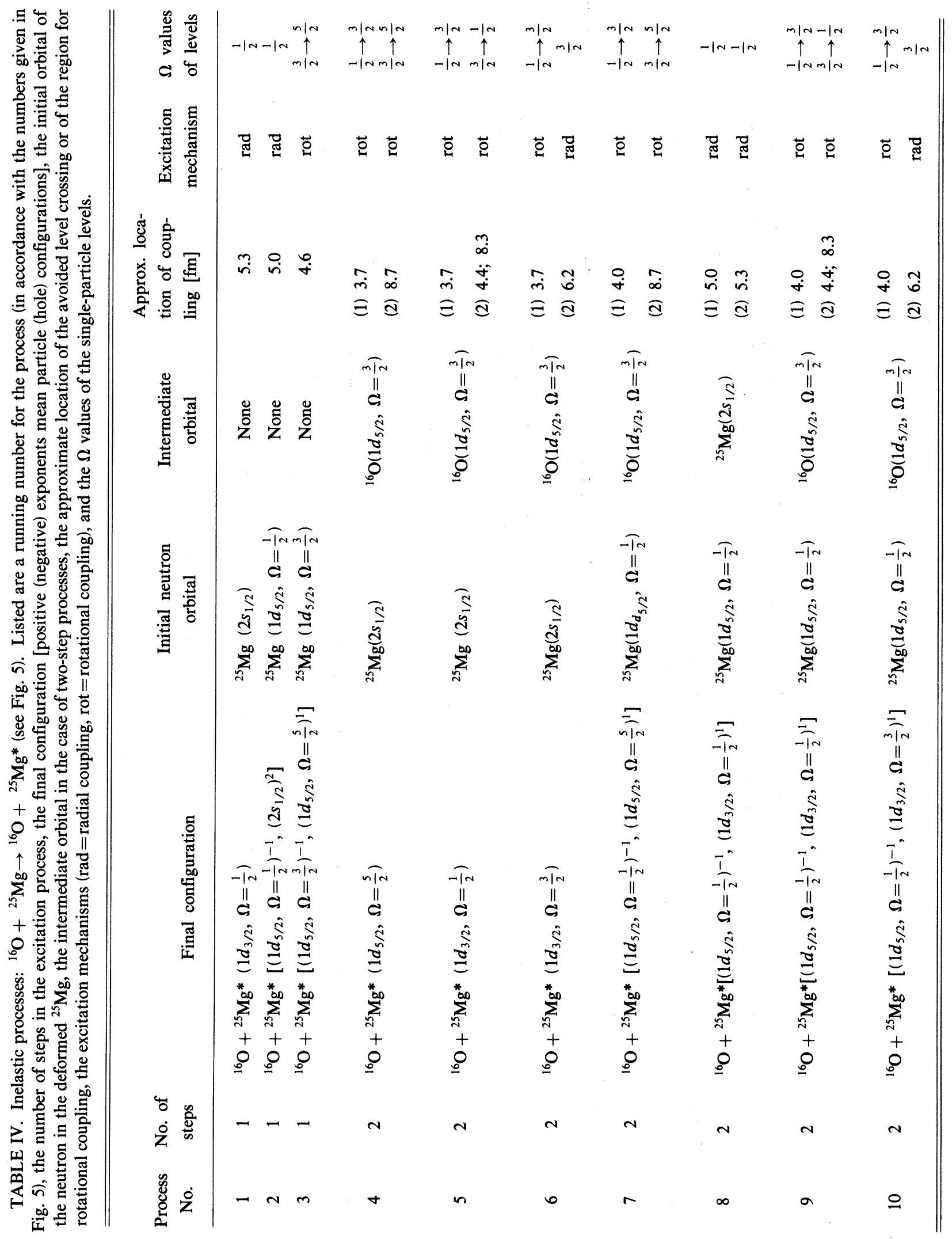




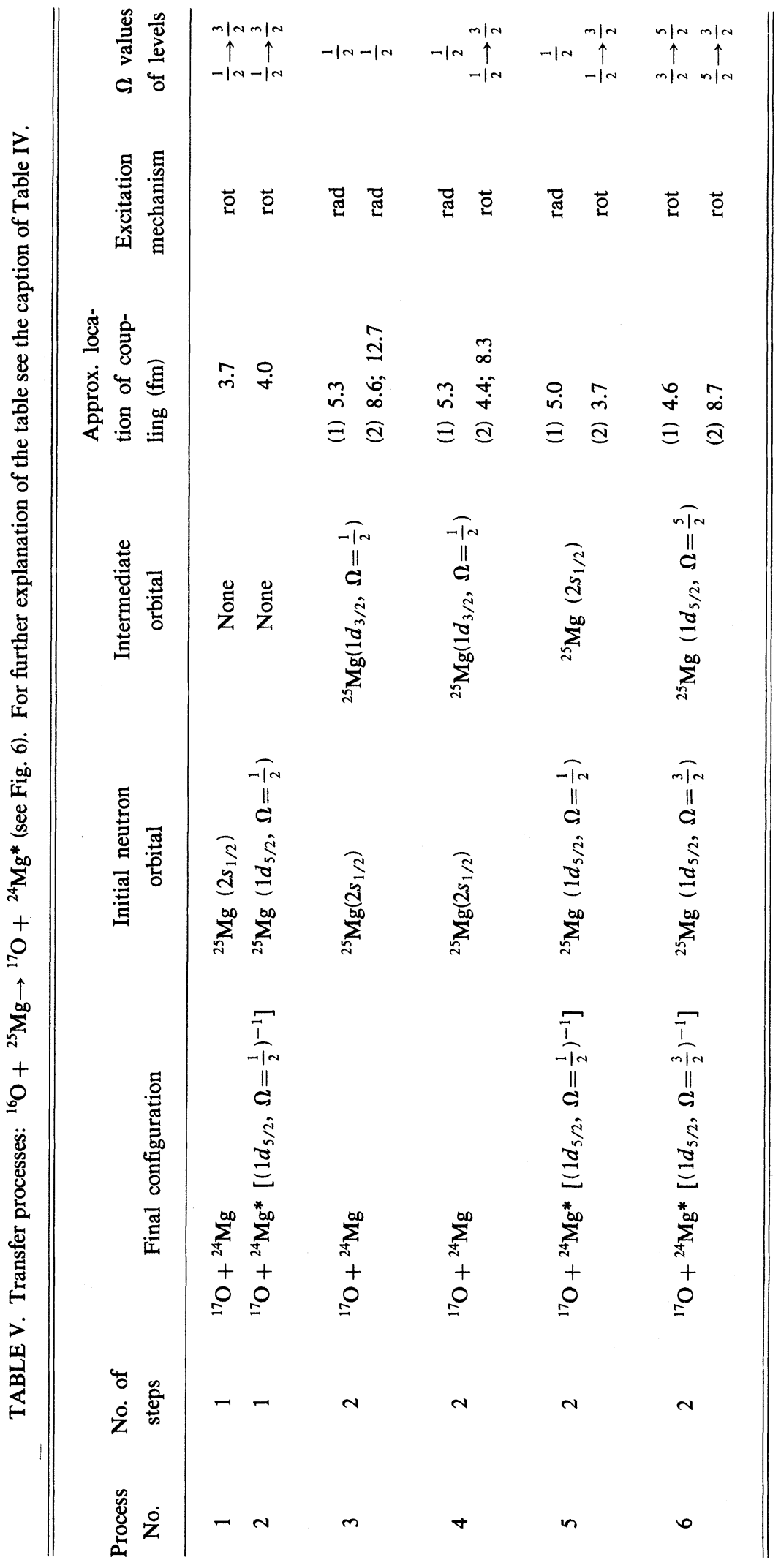


MOLECULAR SINGLE-PARTICLE EXCITATIONS IN HEAVY ...

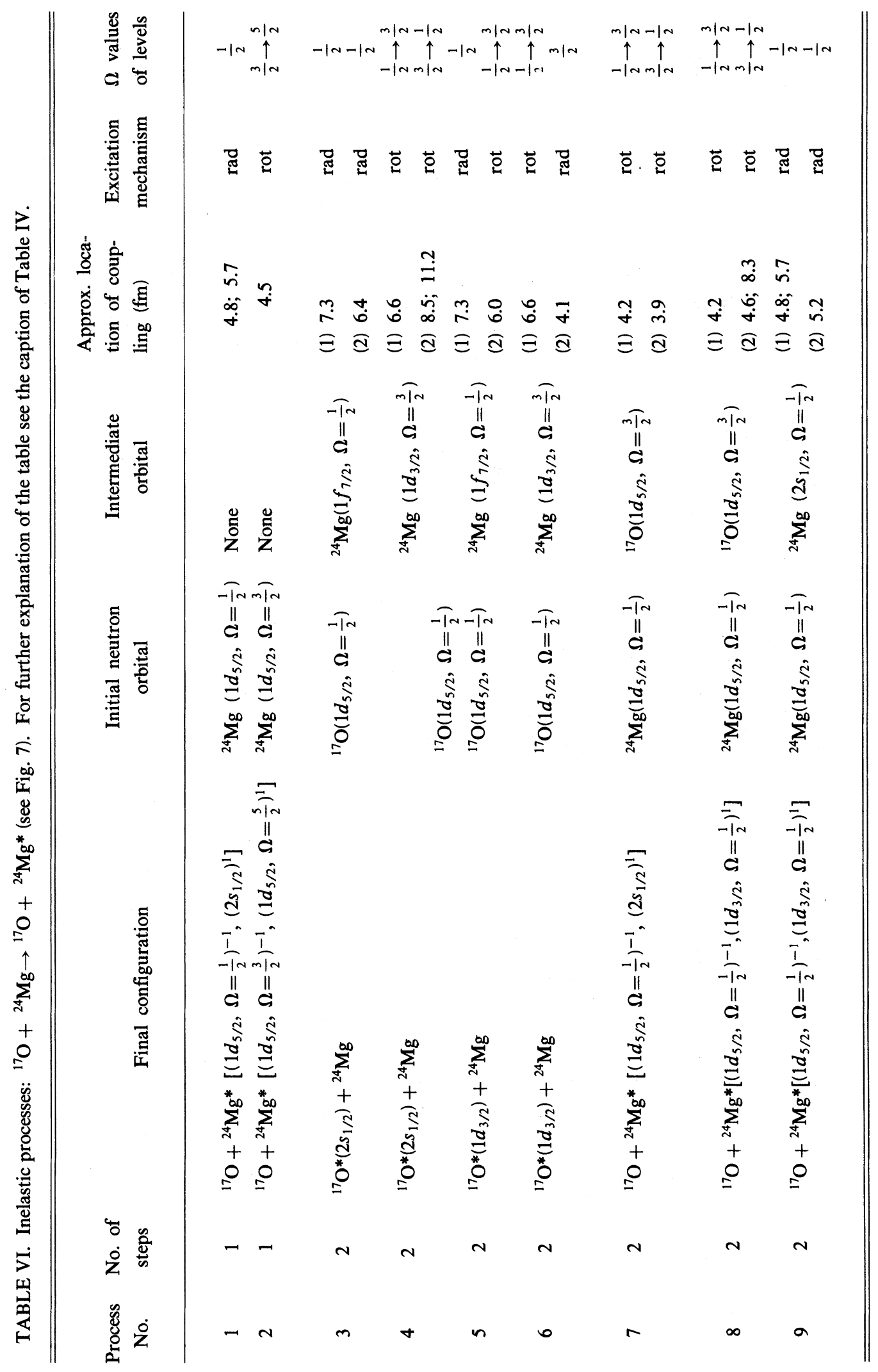




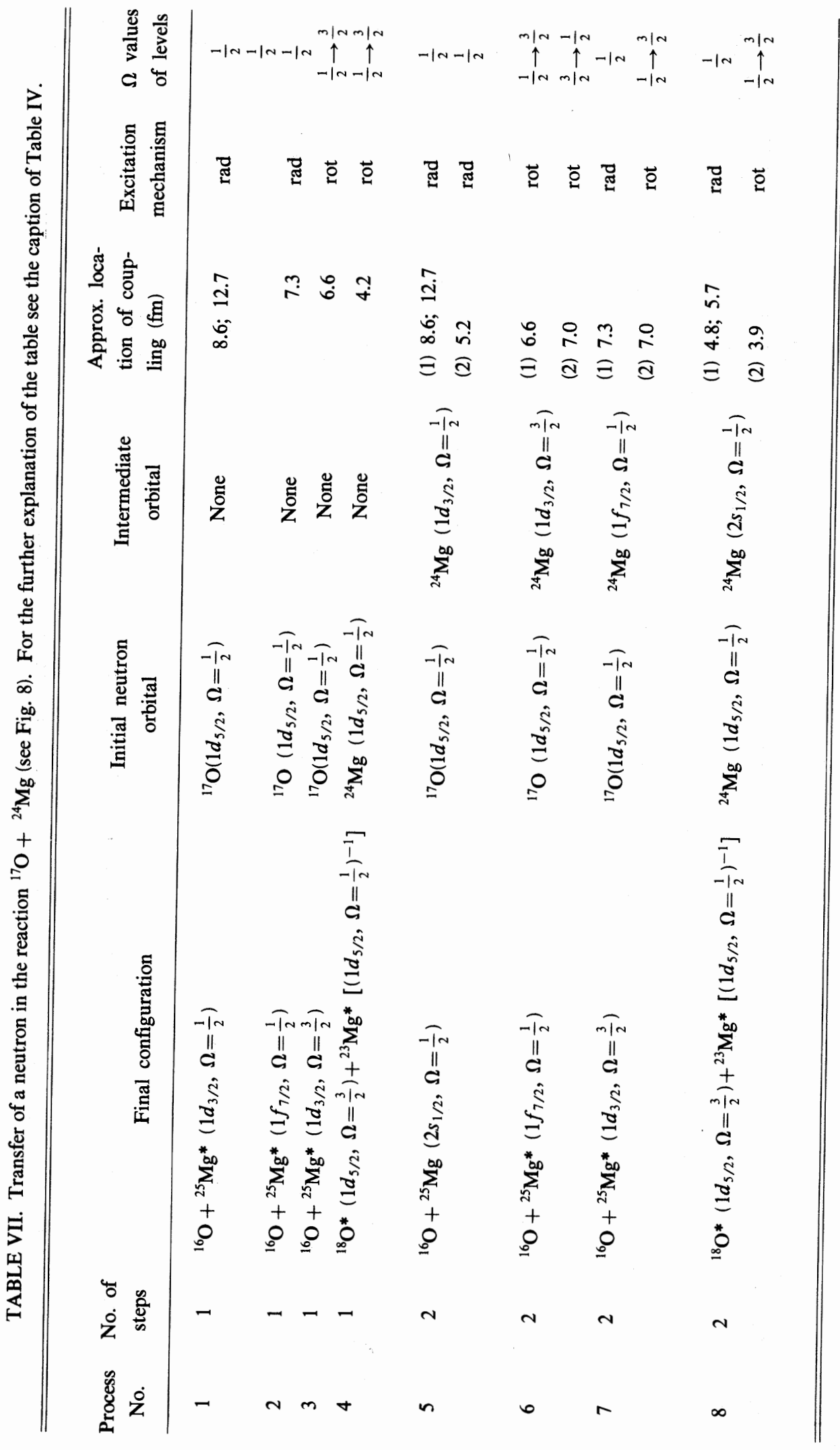


levels involved. In the case of the radial coupling the quantum number $\Omega$ is unchanged, whereas $\Omega$ is changed by one unit by the rotational coupling. The processes are schematically shown in Fig. 5 (inelastic excitation) and Fig. 6 (transfer processes). In these figures the radial coupling at an avoided crossing is indicated by a straight arrow and the rotational coupling by a wavy arrow.

In Fig. 5, for example, we note that the $2 s_{1 / 2}$ level of ${ }^{25} \mathrm{Mg}$, which is occupied initially by the valence neutron, has an avoided crossing with the $1 d_{3 / 2}\left(\Omega=\frac{1}{2}\right)$ level of ${ }^{25} \mathrm{Mg}$ near $5.3 \mathrm{fm}$. This can cause the single-particle excitation to the $1 d_{3 / 2}$ $\left(\Omega=\frac{1}{2}\right)$ state by radial coupling (process number 1$)$. Other inelastic and transfer processes can easily be traced in Figs. $5-8$ by following the corresponding process numbers.

It should be noted in the level diagrams that no level crossings exist for one- and two-step excitations of ${ }^{16} \mathrm{O}$. In all of the listed transfer processes the final ${ }^{17} \mathrm{O}$ nucleus is in its ground state. Furthermore, it is remarkable that not only the loosely bound neutron in the $2 s_{1 / 2}$ level, but also the $1 d_{5 / 2}$ $\left(\Omega=\frac{3}{2}\right)$ and $1 d_{5 / 2}\left(\Omega=\frac{1}{2}\right)$ neutrons, which are bound strongly in the ${ }^{25} \mathrm{Mg}$ nucleus, are involved in these processes. The $1 d_{5 / 2}\left(\Omega=\frac{3}{2}\right)$ and $1 d_{5 / 2}$ $\left(\Omega=\frac{1}{2}\right)$ levels have crossings with unoccupied levels at internuclear distances of 4-5 fm and, therefore, the promotion of these neutrons to excited states can also occur, leading to a particle-hole excitation of ${ }^{25} \mathrm{Mg}$.

\section{B. The ${ }^{17} \mathrm{O}+{ }^{24} \mathrm{Mg}$ reactions}

In the same manner as discussed in Sec. III A one can apply the level diagram shown in Fig. 4 to predict possible inelastic excitations and neutron transfer reactions in the case of the scattering of ${ }^{17} \mathrm{O}$ on ${ }^{24} \mathrm{Mg}$. The processes are summarized in Tables VI and VII and schematically sketched in Figs. 7 and 8.

Among the possible neutron transfers we find two types of processes in which the loosely bound neutron of ${ }^{17} \mathrm{O}$ or one of the neutrons in the $1 d_{5 / 2}$ $\left(\Omega=\frac{1}{2}\right)$ levels of ${ }^{24} \mathrm{Mg}$ is transferred. In the first case the final nuclei are ${ }^{16} \mathrm{O}+{ }^{25} \mathrm{Mg}$, while in the second case they are ${ }^{18} \mathrm{O}+{ }^{23} \mathrm{Mg}$.

In Tables IV-VII, we note that both radial and rotational couplings are equally frequent in these reactions. In general, the radial coupling has a larger strength than the rotational one, as found in the work of Terlecki et al. ${ }^{13}$ Hence we expect that those processes have the largest yields in which the radial coupling is involved. Therefore, the Landau-Zener effect, ${ }^{12}$ inducing the transitions at avoided level crossings, is the most important excitation mechanism for the inelastic excitation and neutron transfer when the reaction is described in terms of molecular single-particle states of a two-center shell model.

\section{CONCLUDING REMARKS}

Realistic two-center level diagrams give us greater microscopic understanding for the selectivity of specific reaction channels in the nucleusnucleus collision and for the role played by the valence nucleons in heavy-ion reactions. Studies, such as the present one, help us to select important inelastic excitation and reaction channels and simplify coupled channel calculations which are tedious and difficult when a complete molecular Hamiltonian is used. The present study also sheds light on the sequence of configurations of the heavy-ion systems during the course of multistep reactions. Since the enhanced excitation at level crossings is distinctly a molecular effect, it is hoped that the measurement of excitation functions for inelastic scattering and neutron transfer reactions for the ${ }^{16} \mathrm{O}+{ }^{25} \mathrm{Mg}$ and ${ }^{17} \mathrm{O}+{ }^{24} \mathrm{Mg}$ systems will give an experimental proof for molecular single-particle orbitals.

Level crossings between an occupied and unoccupied levels can destroy the internal structures, and hence lead to a damping of the elastic scattering process and coupling to the inelastic and transfer channels. Single-particle excitations induced by the nuclear Landau-Zener mechanism ${ }^{12}$ at avoided crossings thus have an influence on the imaginary part of the scattering potential as discussed by Glas and Mosel. ${ }^{14}$ A systematic study of the promotion effects at crossings for various projectile target systems should be done on the basis of realistic twocenter shell models in order to understand microscopically the role of individual molecular nucleonic orbitals in the mechanism of absorption that may produce characteristic signatures in the excitation functions and angular distributions.

\section{ACKNOWLEDGMENTS}

One of us (J. P.) would like to gratefully acknowledge the hospitality of Professor John Blair and Professor Larry Wilets and the stimulating at- 
mosphere at the Topical Research Institute of Theoretical Physics at the University of Washington, Seattle, where a large portion of the present work was carried out. Discussions with many par- ticipants at the Institute were helpful. This work was supported by the Deutsche Forschungsgemeinschaft (DFG) and the Bundesministerium für Forschung und Technologie (BMFT).
${ }^{1}$ Nuclear Molecular Phenomena, Proceedings of the International Conference on Resonances in Heavy Ion Reactions, Hvar, Yugoslavia, 1977, edited by N. Cindro (North-Holland, Amsterdam, 1978); Proceedings of the International Conference on the Resonant Behavior of Heavy-ion Systems, Aegean Sea, Greece 1980, edited by G. Vourvopoulos, Athens, Greece, 1981.

${ }^{2}$ S. Korotky, K. A. Erb, R. L. Phillips, S. J. Willett, and D. A. Bromley, private communications; Bull. Am. Phys. Soc. 24, 13 (1979).

${ }^{3}$ W. von Oertzen, B. Imanishi, H. G. Bohlen, W. Treu, and H. Voit, Phys. Lett. 93B, 21 (1980).

${ }^{4} \mathrm{~B}$. Imanishi, O. Tanimura, and H. Ohnishi, Proceedings of the International Conference on Nuclear Structure, Tokyo, Japan, 1977, J. Phys. Soc. Jpn. 44, Suppl. 257 (1978); Phys. Lett. 57B, 309 (1975).

5J. Y. Park, W. Greiner, and W. Scheid, Phys. Rev. C 21 ,
958 (1980).

6J. Maruhn and W. Greiner, Z. Phys. 251, 431 (1972).

${ }^{7}$ P. M. Endt and C. van der Leun, Nucl. Phys. A310, 1 (1978).

${ }^{8}$ A. E. Litherland, H. McManus, E. B. Paul, D. A. Bromley, and H. E. Gove, Can. J. Phys. $\underline{36}, 378$ (1958).

${ }^{9}$ K. H. Bhatt, Nucl. Phys. 39, 375 (1962).

${ }^{10}$ J. M. Eisenberg and W. Greiner, Nuclear Theory (North-Holland, Amsterdam, 1970), Vol. 1.

${ }^{11} \mathrm{~A}$. Bohr and B. R. Mottelson, Nuclear Structure (Addison-Wesley, Reading, 1975), Vol. 2.

${ }^{12}$ L. Landau, Phys. Z. Sow. 2, 46 (1932); C. Zener, Proc. Soc. (London) A137, 696 (1932).

${ }^{13}$ G. Terlecki, W. Scheid, H. J. Fink", and W. Greiner, Phys. Rev. C 18, 265 (1978).

${ }^{14}$ D. Glas and U. Mosel, Nucl. Phys. A264, 268 (1976).

${ }^{15}$ F. Ajzenberg-Selove, Nucl. Phys. A281, 1 (1977). 